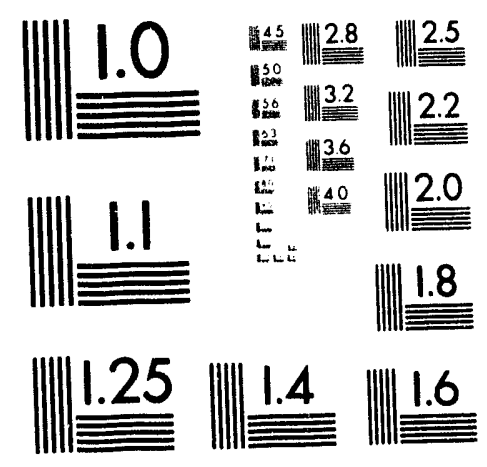



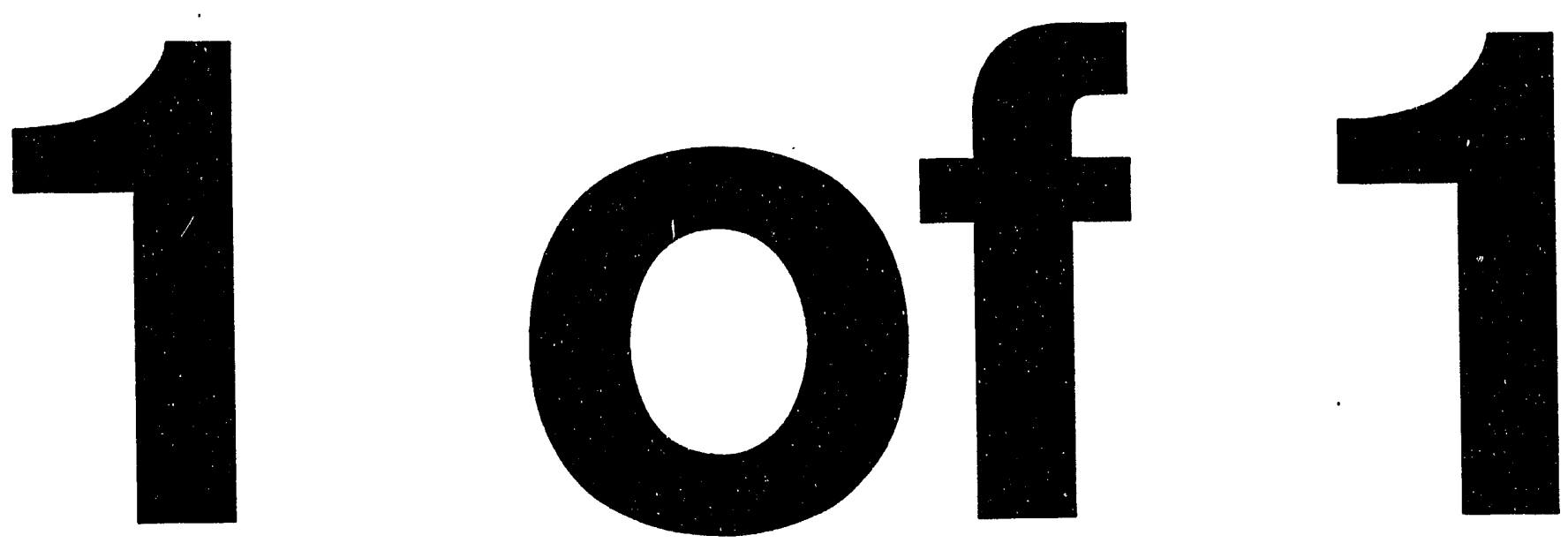


$$
\text { Couf-930269--17 }
$$

UCRL-JC-112246

PREPRINT

\title{
OVERVIEW OF WARP, A PARTICLE CODE FOR HEAVY ION FUSION
}

\author{
Alex Friedman, David P. Grote, Debra A. Callahan, \\ A. Bruce Langdon, and Irving Haber ${ }^{\dagger}$ \\ Lawrence Livermore National Laboratory, University of California \\ P.O. Box 5508, L-440, Livermore California 94550, USA \\ ${ }^{\dagger}$ Naval Research Laboratory \\ Washington DC 20375, USA \\ ifornia
}

This paper was prepared for submittal to the

Proceedings of the Computational Accelerator Physics Conference

Pleasanton, California

February 22-26, 1993

February 22, 1993

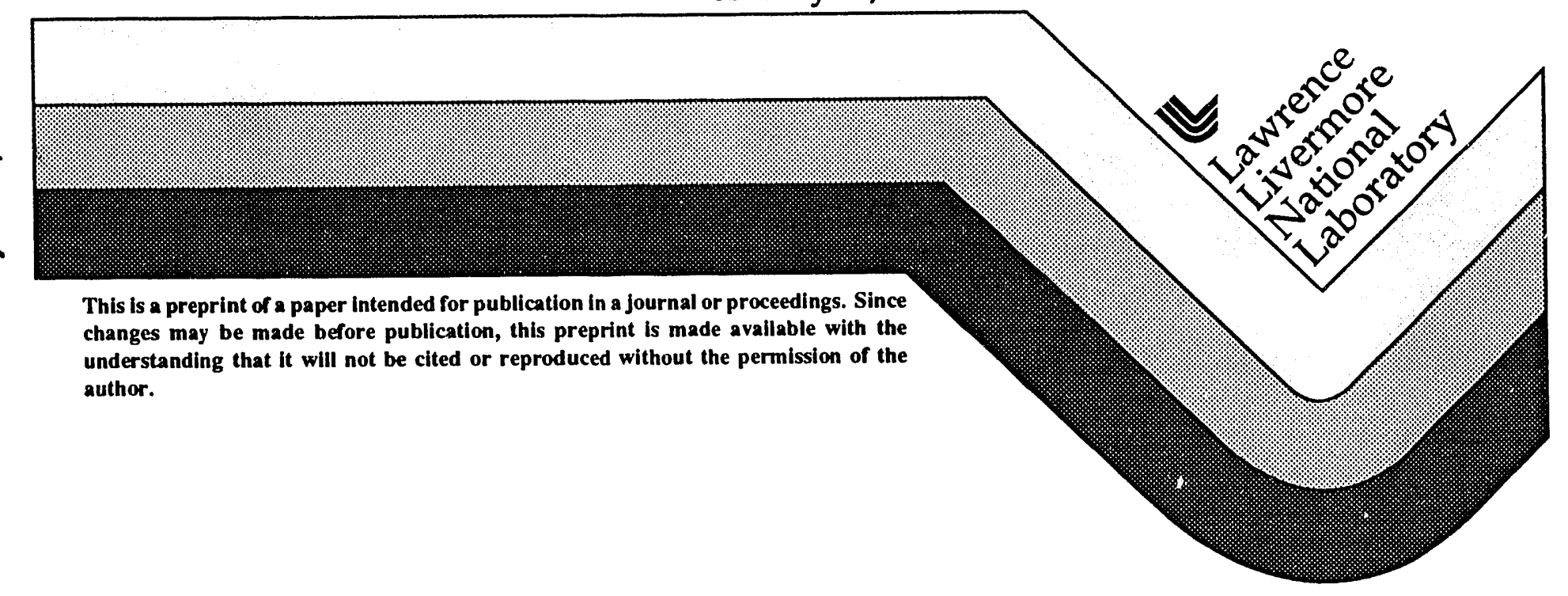

\section{MASTER}

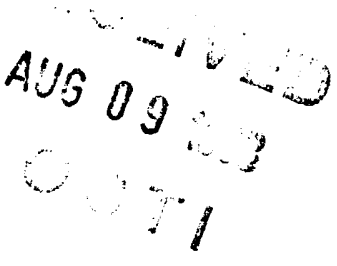




\section{DISCLAIMER}

This document was prepared as an account of work sponsored by an agency of the United States Govermment. Neither the United States Govemment nor the University of Califomia nor any of their employees, makes any warranty, express or implied, or assumes any legal liability or responsibility for the accuracy, completeness, or usefulness of any information, apparatus, product, or process disclosed, or represents that its use would not infringe privately owned rights. Reference herein to any specific commercial products, process, or service by trade name, trademark, manufacturer, or otherwise, does not necessarily constitute or imply its endorsement, recommendation, or favoring by the United States Govemment or the University of Califomia. The views and opinions of authors expressed herein do not necessarily state or reflect those of the United States Govemment or the University of Califomia, and shall not be used for advertising or product endorsement purposes. 


\title{
OVERVIEW OF WARP, A PARTICLE CODE FOR HEAVY ION FUSION*
}

\author{
Alex Friedman, David P. Grote, Debra A. Callahan, and A. Bruce Langdon \\ Lawrence Livermore National Laboratory, Livermore CA 94550 \\ Irving Haber \\ Naval Research Laboratory, Washington DC 20375
}

\begin{abstract}
The beams in a Heavy Ion beam driven inertial Fusion (HIF) accelerator must be focused onto small spots at the fusion target, and so preservation of beam quality is crucial. The nonlinear self-fields of these space-charge-dominated beams can lead to emittance growth; thus a self-consistent field description is necessary. We have developed a multi-dimensional discrete-particle simulation code, WARP, and are using it to study the behavior of HIF beans. The code's $3 \mathrm{~d}$ package combines features of an accelerator code and a particle-in-cell plasma simulation, and can efficiently track beams through many lattice elements and around bends. We have used the code to understand the physics of aggressive drift-compression in the MBE-4 experiment at Lawrence Berkeley Laboratory (LBL). We have applied it to LBL's planned ILSE experiments, to various "recirculator" configurations, and to the study of equilibria and equilibration processes. Applications of the $3 \mathrm{~d}$ package to ESQ injectors, ${ }^{1}$ and of the $r, z$ package to longitudinal stability in driver beams, ${ }^{2}$ are discussed in related papers.
\end{abstract}

\section{INTRODUCTION}

Heavy-ion particle accelerators are attractive candidates as drivers for inertial fusion energy applications. ${ }^{3}$ However, in a fusion driver it is necessary to transport a much larger current than those which have been achieved in existing ion accelerators, and the physics of high-current beams is considerably more complicated than that of the beams in conventional ion accelerators. This is especially true for the recirculating induction accelerator being studied at Lawrence Livermore National Laboratory (LLNL) and at LBL as a lower-cost alternative to a linear driver for fusion energy, ${ }^{4,5}$ because of the extra beam manipulations involved. A variety of numerical tools are employed in the study of HIF beams; ${ }^{6}$ here we describe the WARP discrete-particle simulation effort.

WARP was developed specifically for the study of space-charge-dominated beams. In an HIF driver, such beams must be accelerated and transported over large distances, and undergo a number of manipulations, which may include: transport around bends (needed to enter the target chamber, or for recirculation); transport through imperfectly alioned focusing elements; non-steady acceleration; injection into rings; merging; and splitting.

*This work was performed under the auspices of the U.S. D.O.E. by Lawrence Livermore National Laboratory under contract W-7405-ENG-48, and by the Naval Research Laboratory under contracts DE-AI05-92ER54177 and DE-AI05-83ER40112. 
In earlier stages of progress, the WARP code effort has been described in the Proceedings of the International Symposium on Heavy Ion Inertial Fusion, Dec. 3-6, 1990, ${ }^{7-9}$ and elsewhere. ${ }^{10-12}$ In this paper we briefly review the code concept, methods, and applications. These applications include studies of: beam drift-compression in a misaligned lattice of quadrupole focusing magnets; beam equilibria, and the approach to equilibrium; the MBE-4 experiment recently concluded at $\mathrm{LBL} ;{ }^{13}$ and $3 \mathrm{~d}$ simulations of bent-beam dynamics relevant to planned ILSE experiments. ${ }^{14}$

The code's newest capabilities include a model for an ESQ injector in 3d, using a beam formed "by injection," and an improved $r, z$ package, incorporating a model of module impedances which can drive longitudinal instability. These are described in detail in other papers in these Proceedings. ${ }^{1,2}$

\section{CODE OVERVIEW}

The WARP code contains a number of distinct parts, including: a $3 \mathrm{~d}$ PIC package, called WARP3d, which uses a "warped Cartesian" mesh in $x, y, z$ to describe bends; an axisymmetric $r, z$ PIC package, WARPrz, described elsewhere; $;^{2,9}$ an envelope equation solver (used for loading a "matched" beam); and facilities for initialization, diagnostics, etc. The code uses Basis, ${ }^{15}$ which provides a code development system that facilitates modular construction of programs, and a powerful interactive user interface.

WARP runs in single precision on computers with a 64-bit architecture (Cray C-90, Cray 2, etc.), and in double precision on 32-bit machines (currently Sun and IBM RS-6000; porting to others on which Basis runs will require very minimal effort). As the code runs, graphical output is generated using the NCAR graphics library; a high-level interface to this library provides for double-to-single precision conversion when necessary, as well as a convenient means of generaing multiple plots per page, etc. The code can be run interactively, with the user calling (through the Basis interpreter) for advancement of the particles through one or more timesteps; generation of standard plots, or any other plots thought up on the spur of the moment by the user; production of data dumps (for future restarts or post-processing), and modification of physical and numerical parameters, as the run progresses - a full programming language is available to the user for these purposes. Alternatively, the code can be run to completion in a non-interactive "batch" mode for parameter studies; the same commands which a user might enter at the terminal can be included in one or more input files. Since run times (in 3-D) vary from as little as three minutes (some injector design runs) to tens of hours (runs which look at slow emittance growth phenomena, as for a recirculator), this flexibility is important.

The 3-D code's model accelerator "lattice" consists of a fully general set of finite-length (for the most part, sharp-edged) focusing and bending elements. The electric and magneiic fields of these elements (which have properties such as location, strength, etc. specified by the code's user) are computed algebraically at each particle location at each timestep. In combination with the self-fields, these applied fields are used in the Lorentz force law to advance the particle velocity timestep-by-timestep. Each multipole component (azimuthal harmonic) of the 
applied field is handled separately; for flexibility, different multipoles can overlap axially. The lattice can be made periodic, including periodic alignment errors, to simulate a recirculator or storage ring. Alternatively, the lattice can be periodic, but with aperiodic errors, to simulate a repetitive structure. For efficiency, we load a uniform 1d grid with lattice information (element starts, ends, strengths, etc.) at the beginning of each timestep. When advancing the particles we extract the necessary lattice data from this grid, rather than from the master lists of elements. A typical lattice, in this case one for a possible bend experiment on ILSE, is depicted in Fig. 1.

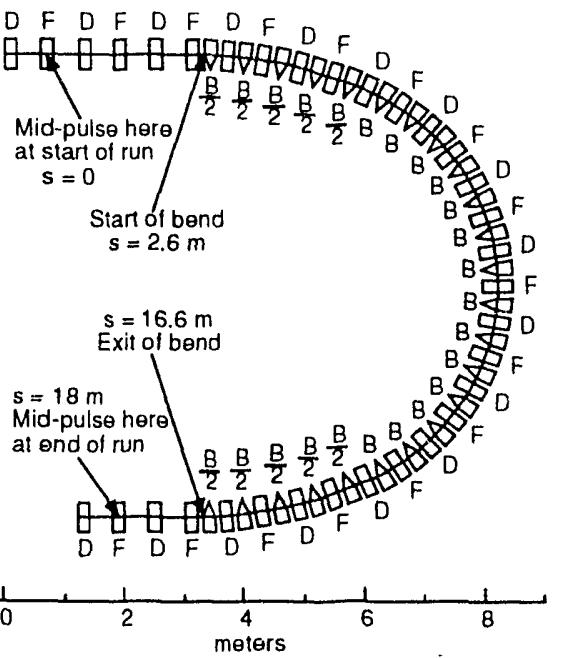

Figure 1: Lattice of focusing and bending elements in $180^{\circ}$ ILSE bend

The simulation takes place in the laboratory frame. The computational mesh fills a moving window and is laid down anew at each timestep. The self-field is assumed electrostatic. The self-potential $\phi$ is obtained via vectorized fast Fourier transform (FFT) operations which assume periodicity in the axial coordinate $z$ but use a Fourier-sine representation in each of the transverse coordinates $x$ and $y$. These are implemented in such a way as to use negligible scratch space. Selffield boundary conditions are most naturally those of a square metal pipe at the transverse $(x, y)$ edges of the mesh. A round pipe, or other shape independent of $z$, can be obtained by use of a $2 \mathrm{~d}$ (transverse) capacity matrix applied independently to each axial Fourier mode. WARPrz's Poisson solver is also based on an FFT in $z$, with tridiagonal matrix inversion in $r$ for each axial Fourier mode.

The fields from electrostatic quadrupoles were originally an idealization, with a perfect sharp-edged axial dependence and only quadrupole and dodecapole terms; this remains an option. Two other models for electrostatic quadrupoles are available. A set of rods, complete with self-field image effects, can be modeled; each group of four rods is handled by its own capacity matrix (the image coupling between rods at different axial locations is small). More recently, electrostatic quadrupoles and ESQ injector structures can be modeled in some generality, with plates attached to the quadrupole elements, holes in the plates through which the beam can pass, etc. This latter model uses an iterative (successive 
over-relaxation) solution. Most recently, the boundary conditions for this solver have been modified to allow subgrid-scale placement of the internal conductor boundaries. Specifically, Poisson's equation at all nodes overlapping a boundary is modified to enforce the condition that the interpolated potential at the intersections of the boundary with each of the three mesh axes has the desired value. This eliminates the "Lego-land" restriction common to particle codes, and allows much coarser meshes to be used. ${ }^{1}$

Usually the entire beam is loaded at the beginning of a run $(t=0)$, with guidance from the envelope solution for initial particle positions and velccities. For injector studies, the beam is formed by injection; particles are continually created along an equipotential surface as time advances.

To model driver-scale beams (which have speeds up to about $c / 3$ ), we plan to use Lorentz transformations (at least in simple straight systems) to obtain the lab-frame self-E and $\mathbf{B}$ needed to advance the particles. We currently use $\mathbf{E}_{\text {self }}$ directly, a good approximation for the slower beams of near-term experiments.

The particle advance is based upon a leapfrog algorithm. However, when plots are to be made, particle moments collected, data dumped, etc., the velocities are advanced through a half-step so that the user sees only "synchronized" positions and velocities. In such a case the velocity advance on the next timestep is also through a half-step. Care is taken in computing particle moments for diagnostic purposes to assure that the transverse moments (e.g., emittance) are not confounded by the phase advance which occurs over the finite-length "window" from within which particles contribute. Scratch copies of the particles' transverse positions and velocities are corrected to the values they would have at the midpoint of the window.

In a numerical calculation of particle trajectories, if a particle were to land within a sharp-edged focusing or bending element on four steps while its neighbor did so on only three, they would receive dramatically different impulses. Thus, the advance is modified to incorporate "residence corrections" for element forces; these corrections multiply the applied field by the fraction of the velocity advance step spent within the element. This technique allows much bigger computational steps than are otherwise possible. ${ }^{10}$

For efficiency on vector compinters such as the Cray C-90, the particle advance is vectorized. Deposition of a particle's contribution to the charge density $\rho$ on the computational mesh is also vectorized, but with a short vector of length eight, depositing "simultaneously" into the eight cells overlapped by each particle. No mesh arrays for the components of $\mathbf{E}$ are used; instead, values of $\phi$ are gathered from 32 cells in the neighborhood of each particle, and then differenced to obtain $\mathbf{E}$ at that particle's location. This saves the space of three $3 \mathrm{~d}$ arrays.

Elongated zones (aspect ratios of order 10:1) have been found to work well provided the axial zone size is less than the beam radius. The timestep size $\Delta t$ is chosen to resolve external field gradients, except at sharp transitions where residence corrections are employed. Relatively fine zoning in the transverse directions is necessary when the beam is strongly tune-depressed. To study slow emittance growth processes reliably, the transverse Debye length should be resolved by the mesh; more rapid, gross phenomena can often be studied using coarser meshes. The plasma period is long $(\mathcal{O} 100 \Delta t)$ and well-resolved. 
We have developed a family of techniques for modeling accelerator bends. These are based upon following a particle's position and velocity in a sequence of rotated inertial Cartesian (laboratory) frames. This "warped" coordinate system is natural for the description of accelerators which include bends. The geometry is depicted in Fig. 2a.
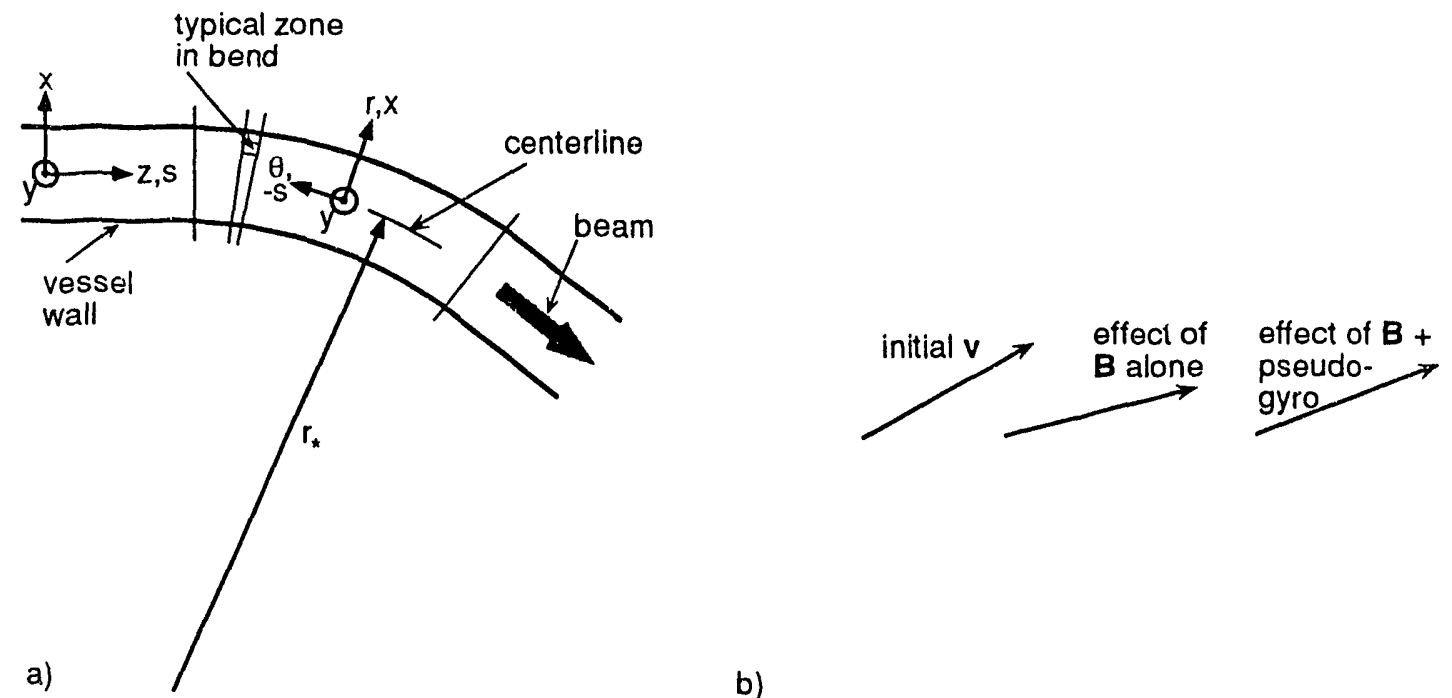

Figure 2: Geometry of the bent-beam algorithm. (a) Simulation domain and coordinate system; (b) effect of folding the pseudogyromotion associated with coordinate rotation into the bending field.

An "exact" method, which is symplectic and independent of aspect ratio, has been described previously, for both $3 \mathrm{~d}$ and $2 \mathrm{~d}$ (transverse) applications ${ }^{12} \mathrm{We}$ have tested that method in a single-particle code, but have not implemented it in WARP; however, a relativistic generalization of it has been successfully applied in the electron accelerator code ELBA. ${ }^{16}$ In that method, a particle is advanced using (e.g.) a leapfrog advance in the coordinate system associated with the particle's location at the beginning of the step. Then, the particle's position and velocity are algebraically transformed into the rotated inertial coordinate system associated with its location at the end of the step. The scheme conserves phasespace area identically, and implies no large-aspect-ratio (gentle bend) expansion. Its numerical properties are those of the underlying difference scheme. The exact method has a non-negligible operation count, but is quite usable. Here, we describe a simpler approximate method now used in WARP3d which is both faster and sufficiently accurate for present purposes. ${ }^{7,10}$

The radius of curvature of the reference orbit (usually the vessel centerline) is $r_{*} \equiv h^{-1}$. Time is the independent variable for particle orbits. The conventional (for accelerator codes) independent variable $s$ is in WARP a dependent variable for orbits, as are $x, y$. In straight sections, $s \equiv z$, while in bends, $s \equiv-r_{*} \theta$. The "radial" coordinate is $x \equiv r-r_{*}$; the unit vectors $\hat{x}$ and $\hat{s}$ evolve as a particle moves, and are different for each particle. The axial speed is $v_{z}=-r \dot{\theta}$ (we use 
subscripts $z$ and $s$ interchangeably). The axial position is advanced in time using:

$$
d s / d t=-r_{*} \dot{\theta}=\left(r_{*} / r\right) v_{z} .
$$

A particle's velociiy vector rotates because of the rotation of the coordinate axes. Due to this: alone, the rate of change of the velocity angle is:

$$
\frac{d}{d t} \arctan \left(\frac{v_{x}}{v_{z}}\right)=-\dot{\theta}=\frac{v_{z}}{r_{*}+x} .
$$

We thus need only augment the dipole (bending) field at each particle position with a "pseudo-gyrofrequency":

$$
B_{y} \Leftarrow B_{y}-\frac{m}{q} \frac{v_{z}}{r_{*}+x}
$$

where $m$ is the particle's mass and $q$ its charge. This folds the necessary backrotation into existing coding. The net effect is shown in Fig. 2b. The algorithm is inexact because $v_{z}$ and $x$ change during the step, but is accurate enough for our needs; "residence corrections" on entry to and exit from bends are necessary.

Poisson's equation in "warped" coordinates is ${ }^{17}$ :

$$
\begin{aligned}
\frac{1}{1+h x} \frac{\partial}{\partial x}\left((1+h x) \frac{\partial \phi}{\partial x}\right) & +\frac{\partial^{2} \phi}{\partial y^{2}} \\
& +\frac{1}{1+h x} \frac{\partial}{\partial s}\left(\frac{1}{1+h x} \frac{\partial \phi}{\partial s}\right)=-4 \pi \rho .
\end{aligned}
$$

Expanding the derivatives, we solve this iteratively. At each iteration the 3d FFT Poisson solver inverts the dominant "Cartesian" second derivative terms. One term, proportional to $(\partial h / \partial s)(\partial \phi / \partial s)$, is included by a simple finite difference, assuming the change in $h$ at bend entry/exit can be spread in $s$ slightly. The iteration converges rapidly, in two or three passes. It is necessary to obtain the true charge density from the "conventional" $\rho_{c}$ collected from the particles, using $\rho=\rho_{c} r_{*} / r$, since (in a bend) the "axial" separation of zones varies with $x$. Also, the axial field is $E_{z}=-\left(r_{*} / r\right) \partial \phi / \partial s$.

\section{SUMMARY OF APPLICATIONS}

Drift Compression: (current enhancement resulting from a head-to-tail velocity gradient or "tilt"): Relatively small misalignments of the focusing quadrupoles can lead to significant off-axis displacements. In'age forces and fringing fields can then induce emittance growth. We seek to learn how fast and how mich the beam may be compressed without unacceptable emittance degradation. The details of the errors, in a system of IJSE scale, are significant; different random-number seeds for the offsets lead to widely varying displacements. ${ }^{11}$

Equilibration: We are examining, in $3 \mathrm{~d}$ and $r, z$, the transfer of thermal energy between transverse and longitudinal motions. For certain ranges of physical parameters, a beam initialized colder in $z$ (axially) than in $x, y$ (transversely) 
is observed to heat rapidly in $z$ until $T_{z}$ is a large fraction of $T_{x, y}$. This appears to be a collective process. ${ }^{11}$

Axial Confinement, Nature of Equilibria: To follow a finite-length beam for a long time, it is necessary to apply an axial confining force. This is done using shaped ends of the accelerating pulses, or "ears." We have modeled (in 3d) near-equilibrium beams that remain "quiescent" over runs as long as 175 lattice periods without significant mid-pulse emittance degradation in the simulation. ${ }^{8}$ The emittance near the tips of the beam does grow. This suggests that the ansatz used in the initial loading, namely a parabolic falloff of line-charge density leading to a cigar-shaped beam with constant phase advance tiroughout the beam and its tips, is not a true equilibrium state. The beam appears to be evolving toward a constant-emittance or constant- $T_{\perp}$ configuration. We are currently exploring these issues further with WARPrz.
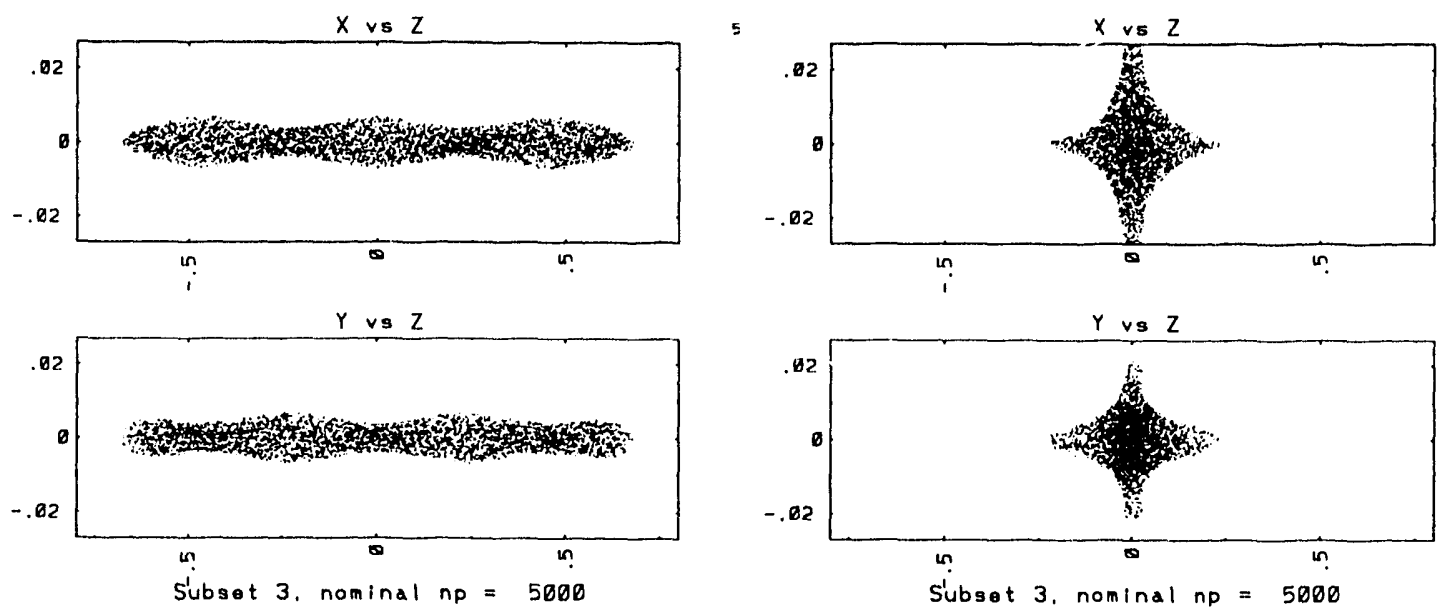

Step $\quad 0 . T=0.0000 \times 10^{-6} \mathrm{~s}$. Zbeam $=0.0000 \mathrm{~m}$

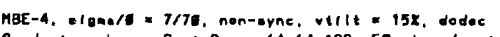

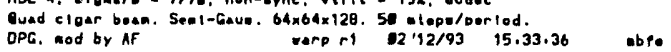

Step 1000. $T=16.6146 \times 10^{-6} \mathrm{~s}$. Zbeam $=9.1440 \mathrm{~m}$

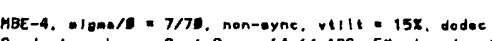

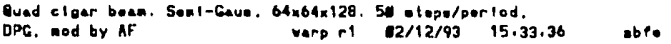

Figure 3: Views of MBE-4 beam: initial on left, at peak compression on right.

Simulations of the MBE-4 Experiment: In this LBL experiment, emittance growth has been observed to accompany aggressive drift compression. Using WARP, we have confirmed that this results from nonlinearities in the focusing fields which are sampled by the particles to a greater extent when the beam grows "fat" as a result of the compression. The dodecapole term is the chief culprit; however, a more realistic representation of the internal conductors as individual cylinders, using the capacity matrix method, showed less emittance growth in cases where the focusing potentials were at $\pm V_{\text {foc }}$ than in cases where they were at 0 and $2 V_{\text {foc }}$. We have conjectured that this is a result of the beam particles "seeing" the pipe, which is at ground potential, while they are in the short drift regions between quadrupoles.

Figure 3 shows the initial beam in one such simulation, in top and side views. After aggressive drift compression has dramatically shortened the beam, it has the form shown in the right half of the figure. By this time some particles have 

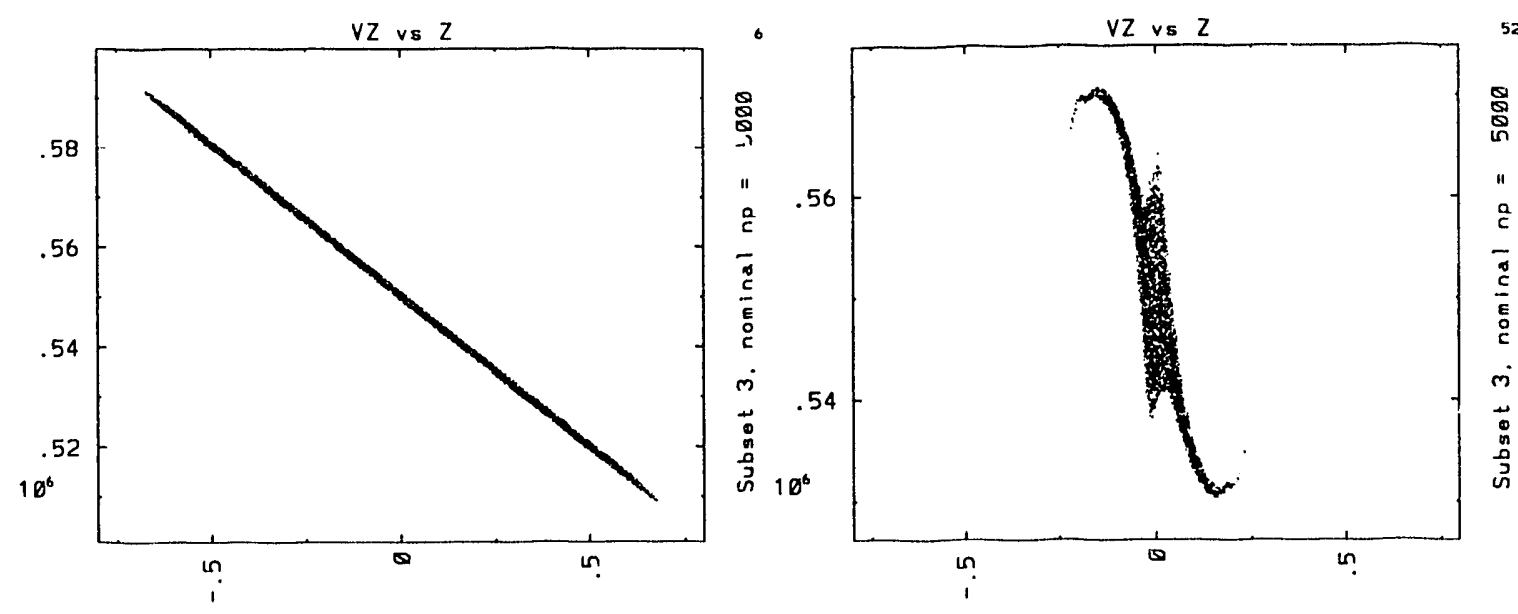

Step $\quad 0 . T=0.0000 \times 10^{-6} \mathrm{~s}$, Zbeam

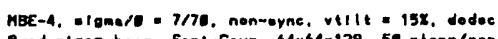

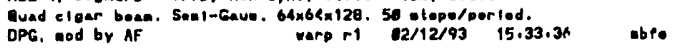

Step 1000, $T=16.6146 \times 10^{-6} \mathrm{~s}$, Zbeam $=9.1440 \mathrm{~m}$

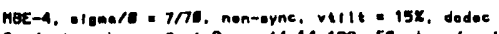

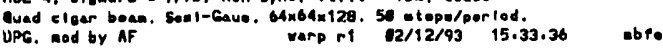

Figure 4: Axial velocity vs. position for MBE-4 beam.

scraped the walls of the chamber and thus have been lost. The actual computer output in diagnostics such as these uses color to indicate different initial axial segments of the beam. Plotted particles retain their color throughout, and so it is possible to discover where different parts of the initial phase space end up. in this run, the inward-directed (in the beam frame) velocity is reduced by the axial self-field, and particles from various axial locations are partly mixed by the end of th: run. Figure 4 shows the $\left(v_{z}, z\right)$ projection of the phase space at the beginning and end of the run.

Longitudinal Stability with Finite Gap Impedance: A known instability is associated with the impedance of the accelerating modules. While careful design and techniques such as feed-forward stabilization should afford suppression of the instability, it is important to be able to model (in a causal, self-consistent way) multidimensional effects such as wave reflection at the bunch ends and radial variations of the interaction between particles and modules. Such effects may be especially significant in the driver-relevant low growth rate regime. We are using WARPrz for these studies. ${ }^{2,9}$

Other applications of the $(r, z)$ model include studies of equilibration processes, of the equilibrium axial dependence of the emittance at the beam ends, and of axial confinement using "ears."

Bent-beam dynamics: We have examined beam behavior in a variety of lattices which incorporate bends; these include models of the $180^{\circ}$ hend planned as an ILSE experiment, and both round and racetrack-shaped recirculator configurations. ${ }^{18}$

A lattice we have considered, similar to one proposed for an ILSE experiment, ${ }^{19}$ is shown in Fig. 1. (These runs simulated a somewhat different bend than is currently planned; modeling of the latest version is underway.) For this system, the phase advances per lattice period are $\sigma_{0}=72^{\circ}, \sigma=20^{\circ}$, and dipoles 
$(20 \mathrm{~cm})$ and quadrupoles $(20 \mathrm{~cm})$ alternate in a FOBODOBO lattice with full period $1.2 \mathrm{~m}$. The first dipole begins at $z=2.6 \mathrm{~m}$, the last ends at $16.6 \mathrm{~m}$ (after $180^{\circ}$ of bending), and we ended the runs at $18 \mathrm{~m}$ (900 steps). We considered axially-cold and -hot $\left(T_{z} \sim T_{\perp}\right)$ beams. The emittance of the axially-hot beam grows; that of the axially-cold beam does not. An axially-hot straight beam in a similar lattice without dipoles does not appear to suffer emittance growth, nor does an emittance-dominated beam in a bend. The beam centroid locations (away from mid-pulse) move radially during their transit of the bend because of the head-to-tail velocity "tilt;" nonetheless, they are re-injected nearly along the centerline of the straight section which follows, due to the "first-order achromat" design.

ESQ injector: We have recently begun modeling this class of systems in $3 \mathrm{~d} .{ }^{1}$ One item of interest is an "energy effect" associated with the fact that the electrode potential differences are comparable to the beam energy, at least at the low-energy end. This leads to some emittance growth, which may be reduced through careful design.

\section{REFERENCES}

${ }^{1}$ D. P. Grote, A. Friedman, and S. S. Yu, these Proceedings.

${ }^{2}$ D. A. Callahan, A. B. Li்ngdon, A. Friedman, and I. Haber, these Proceedings.

${ }^{3}$ T. J. Fessenden and A. Firedman, Nucl. Fusion 31, 1567 (1991).

${ }^{4}$ J. J. Barnard, F. Deadrick, A. Friedman, D. P. Grote, L. V. Griffith, H. C. Kirbie, V. K. Neil, M. A. Newton, A. C. Paul, W. M. Sharp, H. D. Shay, R. O. Bangerter, A. Faltens, C. G. Fong, D. L. Judd, E. P. Lee, L. L. Reginato, S. S. Yu, and T. F. Godlove, "Recirculating Induction Accelerators as Drivers for Heavy Ion Fusion," submitted to Physics of Fluids B, December 1992.

${ }^{5}$ S. S. Yu, J. J. Barnard, G. J. Caporaso, A. Friedman, D. W. Hewett, H. Kirbie, M. A. Newton, V. K. Neil, A. C. Paul, L. L. Reginato, W. M. Sharp, T. F. Godlove, R. O. Bangerter, C. G. Fong, and D. L. Judd, Particle Accelerators 37-8, 489 (1992).

${ }^{6}$ A. Friedman, J. J. Barnard, D. A. Callahan, G. J. Caporaso, Y.-J. Chen, J. F. DeFord, W. M. Fawley, D. P. Grote, I. Haber, K. D. Hahn, E. Henestroza, D. W. Hewett, D. D.-M. Ho, A. B. Langdon, E. P. Lee, A. N. Payne, C. C. Shang, W. M. Sharp, H. D. Shay, L. Smith, and S. S. Yu, "Simulation of Heavy Ion Fusion Beams," to appear in Proc. IAEA Tech. Comm. Mtg. on Advances in Simulation and Modeling of Thermonuclear Plasmas, Montreal, June 15-17, 1992; LLNL Report UCRL-JC-110193, 1992.

${ }^{7}$ A. Friedman, D. P. Grote, D. A. Callahan, A. Bruce Langdon, and I. Haber, Particle Accelerators 37-8, 131 (1992).

${ }^{8}$ D. P. Grote, A. Friedman, and I Haber, ibid., p. 141.

${ }^{9}$ D. A. Callahan, A. B. Langdon, A. Friedman, D. P. Grote, and I. Haber, ibid., p. 97. 
${ }^{10}$ A. Friedman, D. P. Grote, and I. Haber, Phys. Fluids B 4, 2203 (1992).

${ }^{11}$ A. Friedman, R. O. Bangerter, D. A. Callahan, D. P. Grote, A. B. Langdon, and I. Haber, Proc. $2^{\text {nd }}$ European Particle Accelerator Conference, Nice, June 12-16, 1990.

${ }^{12}$ A. Friedman, Proc. $13^{\text {th }}$ Conf. on Numerical Simulation of Plasmas, Santa Fe NM, 1989; LANL, R. J. Mason, Ed.

${ }^{13}$ T. J. Fessenden et. al., Proc. of the 1987 Particle Accelerator Conf., Pp. 898-902, IEEE Cat. No. 87CH2387-9 (1987).

${ }^{14}$ T. Fessenden, R. Bangerter, D. Berners, J. Chew, S. Eylon, A. Faltens, W. Fawley, C. Fong, M. Fong, K. Hahn, E. Henestroza, D. Judd, E. Lee, C. Lionberger, S. Mukherjee, C. Peters, C. Pike, G. Raymond, L. Reginato, H. Rutkowski, P. Siedl, L. Smitl, D. Vanecek, S. Yu, F. Deadrick, A. Friedman, L. Griffith, U. Hewett, M. Newton, and H. Shay, "ILSE, The Next Step toward a Heavy Ion Induction Accelerator for Inertial Fusion Energy," Proc. 14th Int. Conf. on Plasma Physics and Controlled Nuclear Fusion Research, IAEA, Wurzburg, Germany, Sept. 30-Oct. 7, 1992.

${ }^{15}$ P. F. Dubois et. al., "The Basis System," LLNL Document M-225 (1988).

${ }^{16}$ S. Slinker, J. Krall, M. Lampe, and G. Joyce, in Conference Record of the 1991 IEEE Particle Accelerator Conference, San Francisco, edited by L. Lizama and J. Chew (IEEE, NJ, 1991), p. 242.

${ }^{17}$ K. L. Brown and R. V. Servranckx, in Physics of High Energy Particle Accelerators, AIP Conf. Proc. 127, 75 (1983).

${ }^{18}$ J. J. Barnard, H. D. Shay, S. S. Yu, A. Friedman, and D. P. Grote, "Emittance Growth in Heavy Ion Recirculators," Proc. 1992 Linear Accelerator Conference, Ottawa, Ontario, August 24-8, 1992, 229 (AECL-10i28, C. R. Hof.'mann, Ed.).

${ }^{19}$ E. P. Lee, Nucl. Inst. Meth. in Plasma Research A278, 178 (1989). 

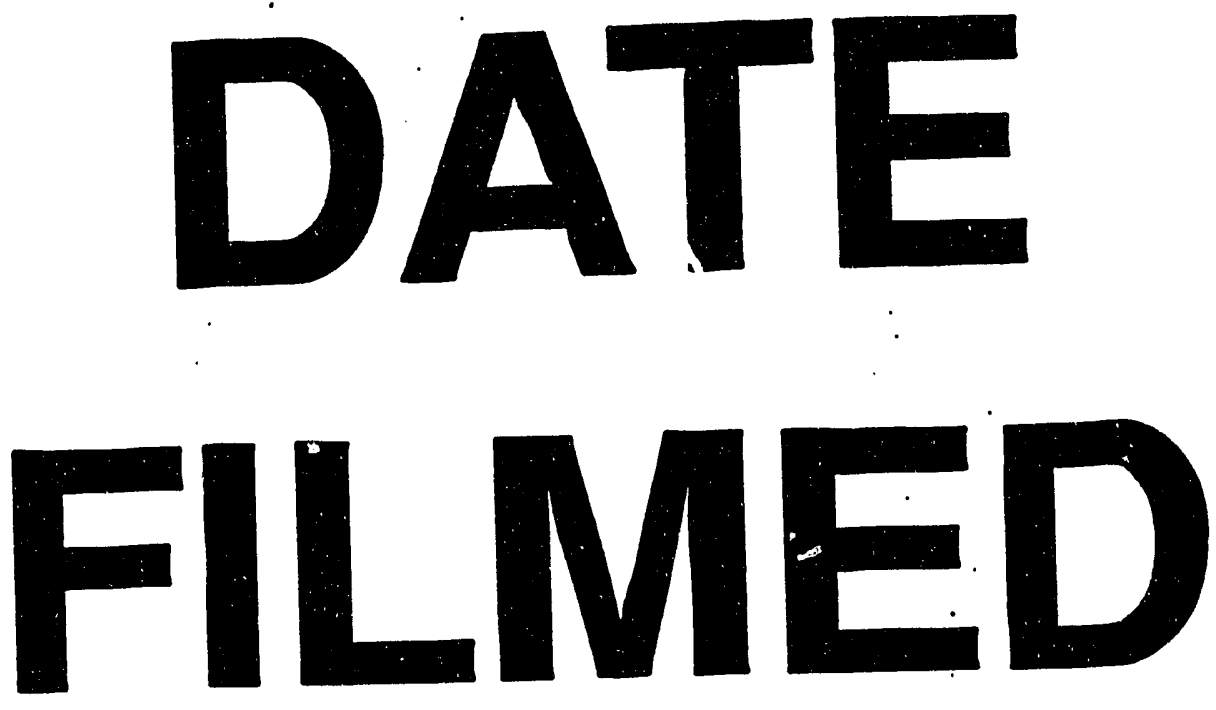

$10 / 19 / 93$
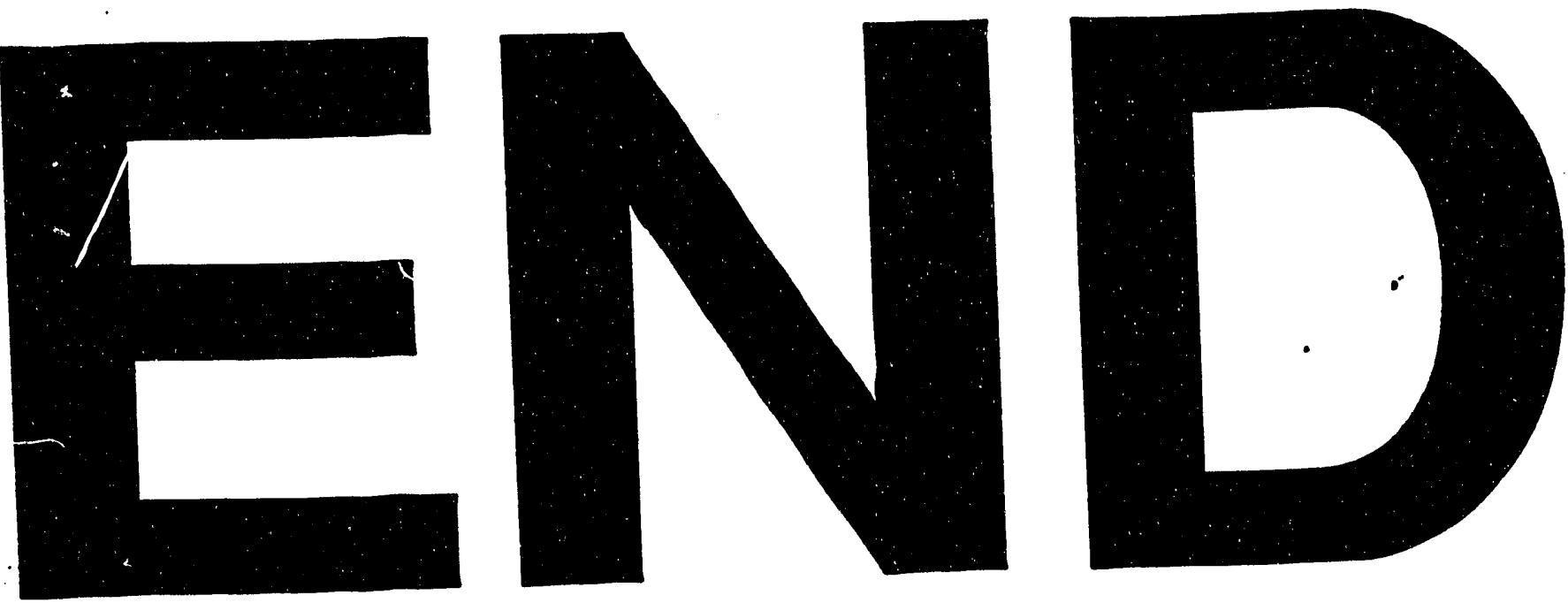
